\title{
Introducción a la fotografía hemisférica en ciencias forestales
}

\section{Introduction to hemispherical photography in forestry}

\author{
Gastón Mauro-Díaz' , José Daniel Lencinas’ y Héctor del Valle²
}

1 Centro de Investigación y Extensión Forestal Andino Patagónico (CIEFAP). Esquel, Chubut, Argentina / Consejo Nacional de Investigaciones Científicas y Técnicas (CONICET), Argentina / gdiaz@ciefap.org.ar

\author{
2 Centro Nacional Patagónico (CENPAT-CONICET). \\ Puerto Madryn, Chubut, Argentina delvalle@cenpat. \\ eduar
}

\section{RESUMEN}

La fotografía hemisférica es profusamente utilizada en climatología y ciencias forestales en vinculación con tecnología geomática. Permite obtener un registro detallado de los claros del dosel y estimar, tanto la estructura del mismo, como la transmisión de luz al sotobosque, ambas variables de gran importancia para el inventario forestal y el manejo de la regeneración natural. Debido a que los equipos de fotografía hemisférica tienen usos muy diversos, incluso en fotografía artística, su costo es bajo en comparación con instrumentos específicos. Para utilizar esta técnica correctamente se requiere de conocimientos particulares de óptica, fotografía y procesamiento digital de imágenes. El objetivo del trabajo es explicar el principio de funcionamiento de la fotografía hemisférica en estimación de parámetros estructurales de bosques. Para ello se discute sobre óptica y fotografía, procesamiento digital de imágenes y estimación de parámetros estructurales. Conocer estos aspectos es relevante para adquirir un equipo, capturar fotografías en el terreno y utilizar softwares específicos para su procesamiento. Las fotografías son archivos raster, los cuales pueden entenderse como una matriz de $n$ filas por $m$ columnas, donde cada celda es un pixel. Para que un raster se visualice como una imagen es necesario establecer un espacio y una profundidad de color. Es importante tener en cuenta que en la captura de la fotografía se producen muchos errores, ya que dependiendo de la región geográfica es muy difícil satisfacer las condiciones ideales de iluminación.

PALABRAS ClAVE: Apertura del dosel, fotografías ojo de pez, fracción de claros, índice de área foliar, procesamiento digital de imágenes.

\section{ABSTRACT}

Hemispherical photography is profusely used in climatology and forest sciences in association with geomatics technology. It is possible to get a detailed record of the canopy and estimate, both of the same structure and understory light transmission, both variables important for forest inventory and management of natural regeneration. Hemispherical photography equipment have very diverse applications, even in artistic photography, and their cost is low compared to specific instruments. Particular knowledge of optics, photography and digital image processing is required to correctly use this technique. The objective of the work is to explain the principle of operation of the hemispherical photography on estimation of structural parameters of forests. This is discussed in terms of optics and photography, digital image processing and estimation of structural parameters. Understanding of these aspects is relevant when it comes to purchasing a computer, capture photographs in the field and use specific software for processing. Photos are raster files, which can be understood as an array of $\mathrm{n}$ rows by $\mathrm{m}$ columns, where each cell is a pixel. So a raster is displayed as an image, it is necessary to establish a color space and color depth. It is important to note that in the capture of the photography major errors occur, depending on the geographical region, because it is very difficult to meet the ideal lighting conditions.

KEY WORDS: Canopy openness, fisheye photography, gap fraction, leaf area index, digital image processing. 


\section{INTRODUCCIÓN}

Los lentes con distancia focal del orden de los $10 \mathrm{~mm}$ (extremadamente corta) y campo visual cercano a los $180^{\circ}$ se conocen como lentes ojo de pez o hemisféricos (Schneider et al., 2009). Desde principios del siglo XX comenzaron a utilizarse en ciencia y técnica (Hill, 1924), actualmente son empleados en climatología y ciencias forestales, muchas veces en vinculación con teledetección satelital y sistemas de información geográfica (Chapman, 2008).

Con fotografías hemisféricas tomadas desde el interior del bosque pueden medirse los claros del dosel (fracción, tamaño y distribución). A partir de esas mediciones es posible estimar estructura y transmisión de luz al sotobosque (Rich, 1990; Hu et al., 2009), ambas de gran importancia para el inventario forestal (Chen et al., 2006; Cruz-leyva et al., 2010) y el manejo de la regeneración natural (Yamamoto, 2000; Yamamoto et al., 2011).

Debido a que los equipos de fotografía hemisférica tienen usos muy diversos, incluso en fotografía artística (Jacobson, 2010), su costo es bajo en comparación con instrumentos específicos (Chen et al., 2006). Aunque no se trate de un instrumento específico, para utilizar esta técnica correctamente se requiere de conocimientos particulares de óptica, fotografía y procesamiento digital de imágenes.

\section{OBJETIVOS}

El objetivo de este trabajo es explicar el principio de funcionamiento de la fotografía hemisférica en estimación de parámetros estructurales de bosques.

El trabajo se divide en los apartados generales Óptica y fotografía, Procesamiento digital y Estimación de parámetros estructurales. El primero trata tanto de la lente y la cámara fotográfica como de los aspectos relacionados con la captura de la imagen en el bosque. En Procesamiento digital se aportan nociones básicas sobre imágenes digitales y procesamiento. En Estimación de parámetros se abordan, mayormente, los principios que sustentan la estimación de parámetros estructurales de primer orden.

\section{ÓPTICA Y FOTOGRAFÍA}

\section{Elementos básicos de una cámara digital}

Una cámara digital está compuesta por el lente, el mecanismo de enfoque, el diafragma, el obturador, el sensor y los comandos para operarla. A través del lente ingresa la luz que llega al sensor, siendo antes regulada por el diafragma y el obturador, los cuales se controlan con la apertura de pupila y la velocidad de obturación, respectivamente. Estas dos variables definen la exposición, que se expresa en la unidad adimensional llamada paso. La velocidad de obturación se mide en segundos, mientras que la apertura se expresa con el número f, el cual es el cociente entre la distancia focal y el diámetro de la pupila de entrada. Por ejemplo, un $\mathrm{f} / 5,6$ indica que la distancia focal es 5,6 veces el diámetro de la pupila (Jacobson, 2010).

El sensor está compuesto por celdas con fotodiodos sensibles al espectro electromagnético visible e infrarrojo cercano. Las celdas se disponen en un arreglo de dos dimensiones, de manera tal que cada una de ellas formará la unidad mínima que compone la imagen digital, el pixel. Para generar imágenes a color, dado que el sensor es monocromático, se coloca un mosaico de filtros para que cada fotodiodo sea estimulado por un solo color. Para que cada pixel tenga información de todos los colores, es necesario un proceso de interpolación conocido como demosaicing (Jacobson, 2010).

La resolución de las cámaras digitales suele expresarse en megapixeles (un millón de pixeles), lo que indica la cantidad de celdas que tiene el sensor y, por consiguiente, la cantidad de pixeles del archivo raster que genera. Sin embargo, la resolución efectiva de las fotografías suele ser un poco menor (Jacobson, 2010).

\section{Función de proyección y de viñeteo}

En fotografía hemisférica se utilizan la proyección equidistante (también llamada polar o equiangular) y la equiángulo sólido (Equisolid-angle). En la primera el ángulo cenital se traslada linealmente en la distancia desde el centro de la fotografía (r), por consiguiente, la distancia entre anillos es constante (figs. 1 y 2). En la equi-ángulo sólido 
las distancias se acortan hacia los bordes del círculo. Estas proyecciones son teóricas, la forma de la lente define el tipo de proyección y ésta suele no ser perfecta, por lo que cada lente tiene una función polinomial de ajuste (Schneider et al., 2009).

El viñeteo es un efecto geométrico-mecánico que oscurece los bordes de la imagen, siendo la función de viñeteo aquella que relaciona atenuación de luz con el ángulo cenital (Lang et al., 2010; Jacobson, 2010).

\section{Tipos de equipo}

Las cámaras fotográficas se dividen en compactas y réflex, diferenciándose en que solamente esta última tiene lentes (objetivos) intercambiables (Jacobson, 2010). Para que las cámaras compactas se conviertan en equipos hemisféricos se les debe acoplar un convertidor, en cambio, a las réflex se les debe cambiar el objetivo. Sin duda, la cámara compacta Nikon Coolpix ha sido el equipo más utilizado; sin embargo, ésta se encuentra fuera de producción (Chapman, 2008).

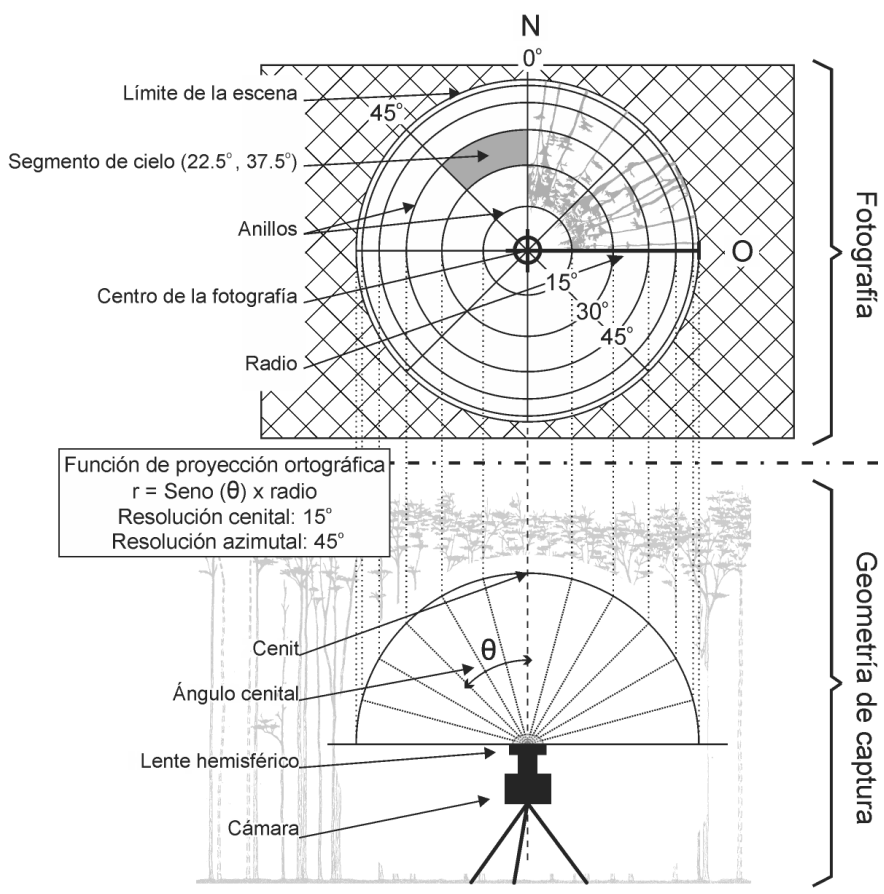

FIGURA 1. Esquema del proceso de captura de una fotografía hemisférica. La función de proyección es meramente ilustrativa ya que no se utilizan lentes de ese tipo (Schneider et al., 2009).
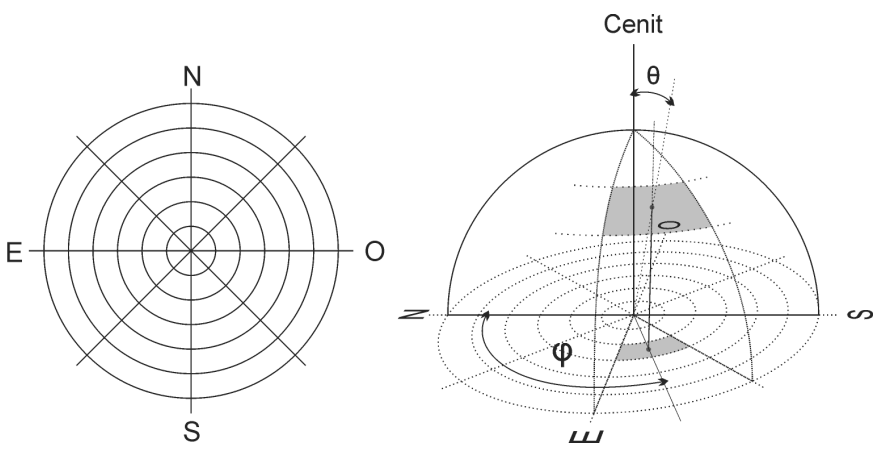

FIGURA 2. Resolución cenital y azimutal para la segmentación del hemisferio en segmentos de cielo. Adaptado de Walter (2011).

Actualmente se utilizan equipos réflex (Macfarlane et al., 2007; Pekin y Macfarlane, 2009), lo que representa un cambio sustancial en el tipo de imágenes que se captura. Mientras que las Coolpix (proyección polar) toman fotografías hemisféricas circulares, las réflex (proyección equiángulo sólido) toman $90^{\circ}$ de ángulo cenital solamente en las 4 esquinas de la fotografía (Fig. 3).
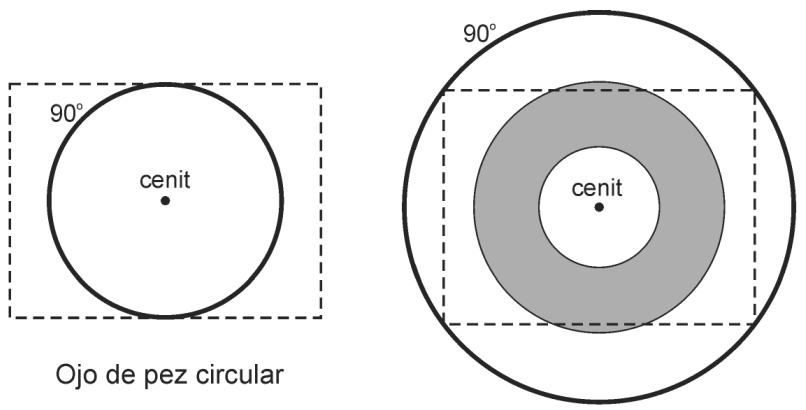

Ojo de pez de fotograma completo

\begin{tabular}{ll|l}
$\begin{array}{l}\text { Proyección completa } \\
\text { del hemisferio }\end{array}$ & Fotografía & $\begin{array}{l}30-60^{\circ} \text { con proyección } \\
\text { equi-ángulo sólido }\end{array}$
\end{tabular}

FIGURA 3. Tipos de fotografías hemisféricas. En la imagen de la izquierda se representa un anillo entre 30 y 60 grados de ángulo cenital asumiendo una proyección equi-ángulo sólido. 

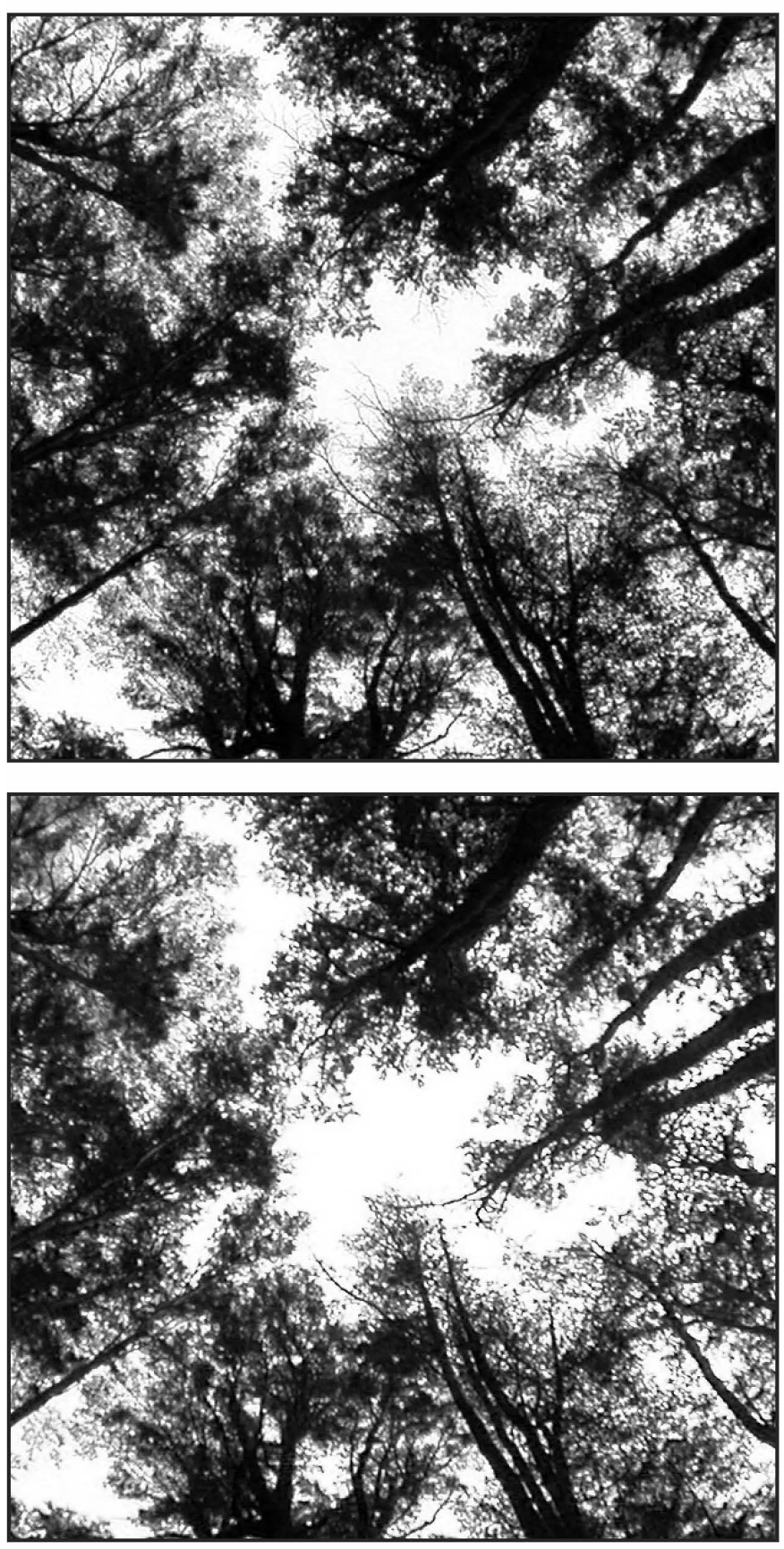

FIGURA 4. Imagen sin (arriba) y con (abajo) efecto blooming (Equipo Nikon Coolpix 5700 / FC-E9, banda del azul, bosque de latifoliadas).

\section{Captura de la fotografía}

Es esencial que la cámara esté nivelada y en un soporte firme. Para ciertas estimaciones y correcciones es necesario orientarla hacia algún punto cardinal de referencia, como, por ejemplo, dirigir la parte superior de la foto hacia el norte.

Para la clasificación digital mediante un valor umbral (se explica en el próximo apartado) es necesario que el registro del cielo sea muy claro y el de la vegetación muy oscuro. Sin embargo, esto tiene que ocurrir con un mínimo de saturación (sobreexposición), porque de lo contrario se produce un efecto conocido como blooming (Fig. 4), que hace ver los claros del dosel más grandes de lo que realmente son (error de sobreestimación). Para obtener el máximo contraste con el mínimo blooming es necesario que el cielo brille homogéneamente, lo cual sucede en días completamente nublados y, en menor medida, en el crepúsculo (Jacobson, 2010; Leblanc et al., 2005; Zhang et al., 2005). Cabe mencionar que la frecuencia de días completamente nublados y estables varía regionalmente, por lo tanto, satisfacer este requerimiento será más difícil en algunos lugares que en otros. Pero no es suficiente con esperar las mejores condiciones de iluminación, todavía resta definir la exposición de la cámara, que afectará tanto al contraste como al blooming.

Las cámaras digitales tienen un modo automático para fijar la exposición que responde a una función interna de la cámara, la cual es regulada por un fotómetro y, por lo tanto, es dependiente del nivel de luz bajo el dosel. En Zhang et al. (2005) se propone un protocolo para fijar la exposición en forma independiente (Fig. 5). Para ello, se debe aumentar la exposición en dos pasos relativos a la exposición determinada automáticamente por la cámara en un claro extenso o directamente bajo el cielo. Este proceso debe repetirse cada vez que las condiciones de iluminación cambien, lo que sucede muy rápidamente en el crepúsculo. Para aumentar la exposición se puede variar la apertura o la velocidad de obturación, pero debe tenerse cuidado con bajas velocidades de obturación porque producen imágenes de poca nitidez debido a las vibraciones de la cámara y el movimiento del follaje. 


\section{PROCESAMIENTO DIGITAL}

\section{La imagen digital}

Las fotografías son archivos raster, los cuales pueden entenderse como una matriz de $n$ filas por m columnas, donde cada celda es un pixel. Éste se trata de un valor digital o varios, con la restricción de que todos los pixeles del raster deben tener igual cantidad de valores digitales, siendo cada capa de valores una banda (Jacobson, 2010). En términos matemáticos, el pixel puede entenderse como un vector de $i$ números, donde $i$ es la cantidad de capas que tiene el raster. Para mayor información, en http:// homepages.inf.ed.ac.uk/rbf/HIPR2/hipr_top.htm se encontrará acceso libre a explicaciones multimedia interactivas relacionadas a los temas de este apartado.

Para que un raster se visualice como una imagen es necesario establecer un espacio de color y una profundidad de color. Los espacios de color comúnmente utilizados en fotografía hemisférica digital son "blanco y negro", “escala de grises” y RGB (siglas del inglés red, green, blue). La profundidad de color indica el rango numérico que tendrá el valor de cada banda en el pixel, las más usuales son 1-bit, 8-bit y 16-bit (Jacobson, 2010; Leblanc et al., 2005). Por ejemplo, un raster con una banda de 8 -bit tendrá $2^{8}$ niveles de valor digital en cada pixel (representado con un número entre 0 y 255), y podrá ser visualizado en escala de grises ( 0 será negro, 255 blanco y los restantes valores gradaciones del gris).

Si bien los fotodiodos responden linealmente a la intensidad de luz, las cámaras digitales no hacen una conversión lineal de la señal en el valor digital. Esto se conoce como corrección gamma, siendo su fin simular la respuesta no lineal del ojo humano. La corrección se trata de una transformación logarítmica que aclara los tonos medios con la ecuación (1).

$$
V D=V D_{\text {max. }} .\left(\frac{B}{B_{\text {max. }}}\right)^{\frac{1}{\gamma}}
$$

Donde VD es el valor digital, $B$ es el brillo del objeto y $\gamma$ es el valor gamma, el cual típicamente oscila entre 2 y 2,5 (Cescatti, 2007).
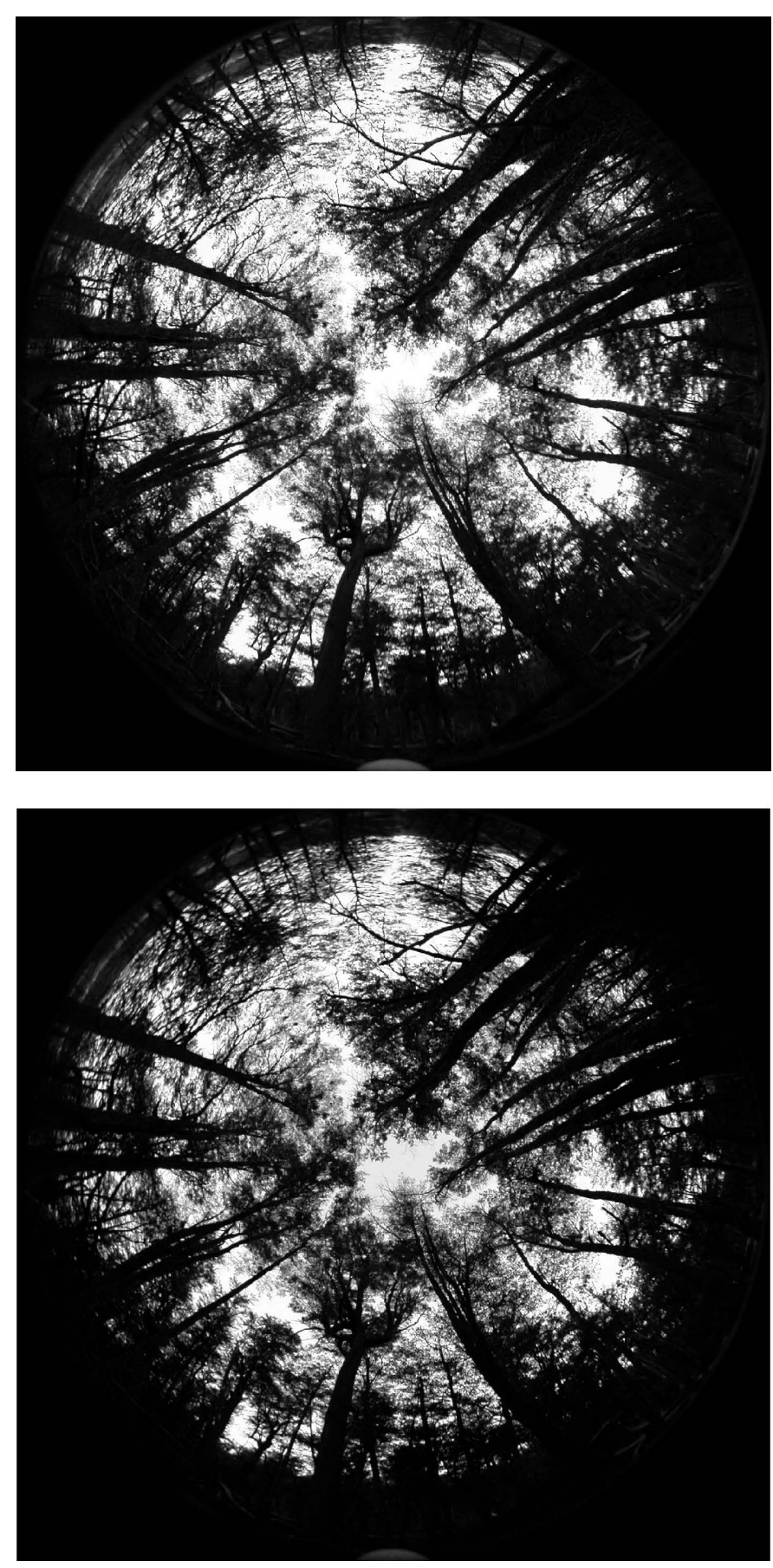

FIGURA 5. Efecto de la exposición sobre la calidad de la fotografía hemisférica (equipo Nikon Coolpix 5700 / FC-E9, banda del azul, bosque de latifoliadas). Fotografías tomadas desde el mismo punto y casi al mismo tiempo, pero con diferente exposición. La de arriba con exposición automática y la de abajo con exposición determinada con el protocolo de Zhang et al. (2005). 

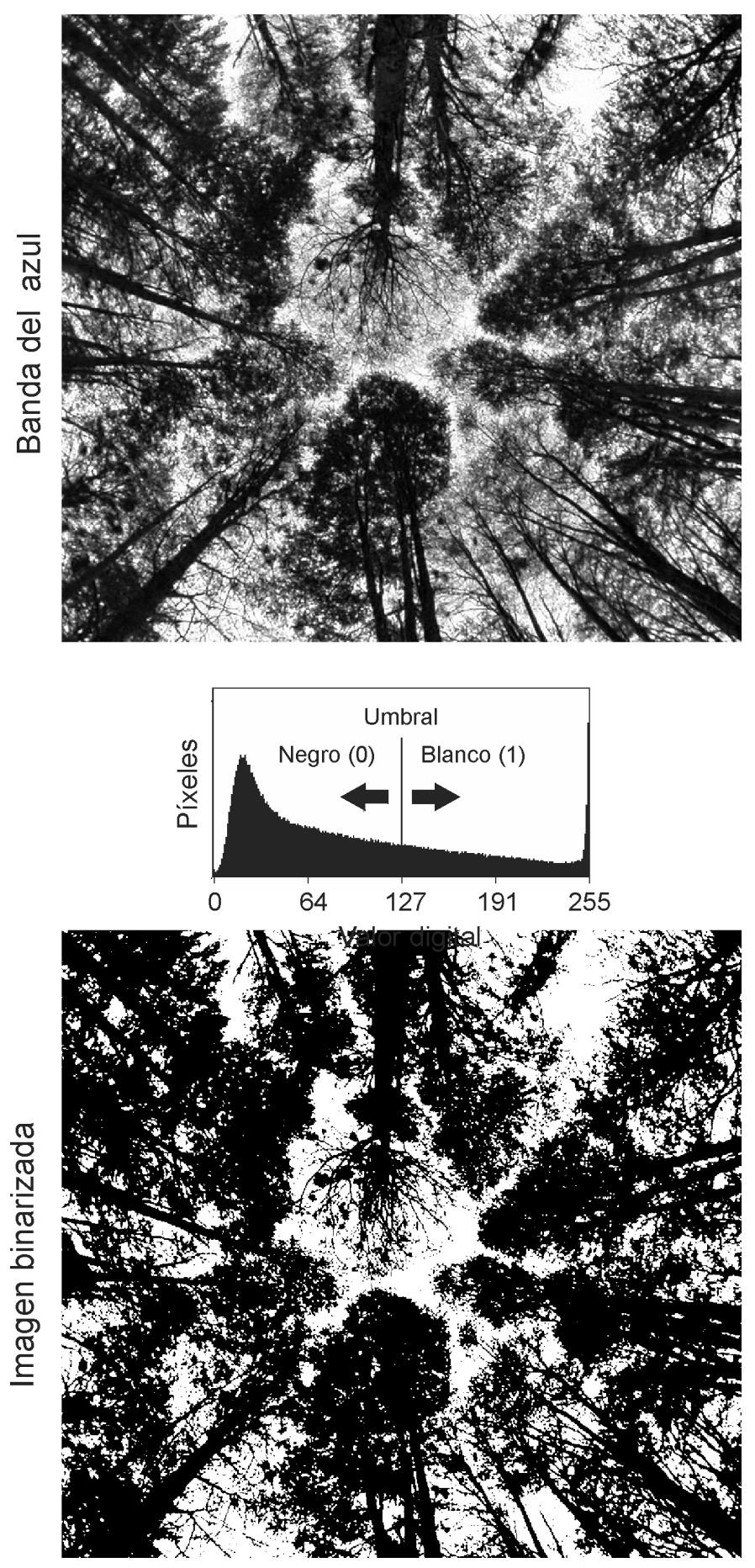

Figur a 6. Binarización de la banda del azul de una fotografía hemisférica (bosque de latifoliadas) mediante un valor umbral.
El formato jpg es un archivo raster con tres bandas de 8-bit (24-bit) preparado para RGB, aunque es posible alterar su espacio de color, por ejemplo, visualizando sólo la banda del azul (blue) en escala de grises. Las cámaras fotográficas permiten almacenar en memoria la imagen en bruto (raw), logrando mayor profundidad de color que el jpg (Lang et al., 2010).

Con relación a la formación del color en una fotografía, aquí se ha dicho que cada fotodiodo (asociado a un pixel) es estimulado por un solo color y a través de una interpolación con los pixeles vecinos (demosaicing) es que se generan las tres bandas RGB. En lentes en general y en lentes hemisféricos en particular, se observan imperfecciones en el color conocidas como aberraciones cromáticas. Éstas se deben a que el índice de refracción de la lente depende de la longitud de onda. Tanto este fenómeno óptico como el demosaicing dan lugar a la manifestación de la aberración cromática, que se observa en los ángulos cenitales mayores y se trata de una baja correspondencia espacial entre bandas (Van-den-Heuvel et al., 2006; Jacobson, 2010).

\section{Clasificación digital}

La técnica de clasificación digital actualmente aplicada a fotografías hemisféricas es simple, pero adecuada para discriminar dos clases con alto contraste. El objetivo de la clasificación es separar los claros de la vegetación, por lo que cada pixel se clasifica en "cielo" y "no-cielo". Como explicamos en Captura de la fotografía, se obtienen imágenes de alto contraste a color, sin embargo, la información de color es muy reducida ya que el cielo es muy brillante y la vegetación muy oscura. Por lo tanto, se dispone de tres bandas con información similar. Como en el espectro electromagnético del azul la vegetación tiene el brillo más bajo (Chapman, 2008), se utiliza solamente la banda del azul (Leblanc et al., 2005).

La clasificación mediante valores umbrales sirve para separar una banda en dos o más clases. Esencialmente consiste en segmentar la profundidad de color a través de valores de corte. El histograma de los valores digitales (profundidad de color) de una imagen monocromática 
(una sola banda) de alto contraste puede separarse con cierta objetividad en dos grupos de pixeles con base en su valor digital. La separación en grupos se hará efectiva cuando a los pixeles con valor digital menor al umbral se le asigne el valor $0 \mathrm{y}$ a los restantes 1 . Este procesamiento digital se conoce como binarización porque genera archivos raster de 1-bit (Ridler y Calvard, 1978).

Cuando se binariza la banda del azul de una fotografía hemisférica de alto contraste (Fig. 6), se le asigna el valor 0 a la vegetación (no-cielo) y 1 a los claros (cielo).

\section{Estimación de parámetros estructurales del dosel}

La estructura del dosel hace referencia a la disposición espacial, superficie y forma de los órganos aéreos (Norman y Campbell, 1989). Puede ser caracterizada por numerosas variables (parámetros estructurales), como la biomasa aérea total, la distribución angular de las hojas y el índice de área foliar (LAI, por sus siglas en inglés), siendo algunas de ellas estimables con fotografía hemisférica (Chapman, 2008).

Mediante la fotografía hemisférica se obtiene un registro detallado de la distribución espacial, tamaño y forma de los claros del dosel (canopy gap) en función del ángulo cenital. Todos los parámetros estructurales relacionados directamente con los claros pueden ser considerados de primer orden, mientras que los obtenidos a partir de estos mediante adopción de supuestos podrán ser considerados de segundo orden (Gonsamo et al., 2010).

El parámetro de primer orden de mayor importancia es la fracción de claros (GF). Se define como el porcentaje de cielo que puede ser visto desde debajo del dosel arbóreo (Chen et al., 1997). Éste puede ser medido con la fotografía hemisférica bajo el supuesto de que los elementos del dosel se comportan como objetos oscuros (i.e. no transmiten luz) en la banda del azul (Gonsamo et al., 2010). Se calcula con las ecuaciones (2) y (3).

$$
\begin{gathered}
G F(\varphi, \theta)=\frac{P_{B}}{P_{N}+P_{B}} \\
G F_{\text {total }}=\frac{1}{N} \sum_{1}^{N} G F(\varphi, \theta)
\end{gathered}
$$

Donde $\varphi$ es el ángulo azimutal medio y $\theta$ es el ángulo cenital medio (ambos de un segmento del hemisferio proyectado en el plano), $P_{N}$ es el número de pixeles negros y $P_{B}$ es el número de pixeles blancos (ambos referidos al segmento del hemisferio $[\varphi, \theta])$ y $N$ es el número de segmentos.

La apertura del dosel (canopy openness, CO) y su complementario, la cobertura del dosel (canopy cover o closure), también son parámetros de primer orden. En este caso se introduce un término que reproyecta la imagen al hemisferio y así se corrige el efecto distorsivo de proyectar el hemisferio en el plano. Se calcula con la ecuación (4).

$$
C O=\sum_{1}^{N} G F(\varphi, \theta) \frac{\cos \theta_{1}-\cos \theta_{2}}{N}
$$

Donde $\theta_{1}$ y $\theta_{2}$ son el menor y mayor ángulo de un segmento del hemisferio y $\mathrm{N}$ es el número de segmentos.

Puede ser oportuno aclarar que este concepto no es equivalente al habitualmente utilizado en ciencias forestales, el cual se refiere a la proyección vertical de las copas sobre el plano horizontal (Walter, 2011).

Existe una gran difusión de software para estimar GF, CO, LAI y transmisión de luz al sotobosque, como el CIMES (http://jmnw.free.fr), el GLA (http://www.ecostudies.org/gla) y el Hemisfer (http://www.wsl.ch/dienstleistungen /hemisfer). Cuando se utiliza este tipo de software, se debe definir la resolución de ángulo cenital y azimutal (Fig. 2), lo que determina el número de segmentos de cielo. En teoría, cuanto mayor sea el número de segmentos mayor será la precisión de las estimaciones. Sin embargo, el LAI es proporcional al - $\mathrm{Ln}(\mathrm{GF})$ y éste no tiene solución para $\mathrm{GF}=0$, por lo tanto, la resolución angular debe ser suficientemente gruesa como para que ningún segmento de cielo quede solamente con pixeles negros. Como ha sido indicado en numerosos estudios, este aspecto del procesamiento tiene un efecto significativo en la determinación del LAI (Gonsamo et al., 2010).

\section{CONCLUSIÓN}

Las posibilidades de aplicar la fotografía hemisférica en ciencias forestales son bastas y se sustentan en el creciente 
desarrollo de la tecnología digital. Sin embargo, antes de utilizar esta técnica se debe conocer su principio de funcionamiento, ya que pueden cometerse errores graves en muchas instancias del procedimiento.

Es posible que en la captura de la fotografía se produzcan los mayores errores ya que, dependiendo de la región, es muy difícil satisfacer las condiciones ideales de iluminación. Los software específicos para procesar fotografías hemisféricas ofrecen numerosas alternativas de procesamiento, las cuales tienen un significativo impacto en la determinación del parámetro de interés. Se espera que este documento sirva para introducir al lector en la materia, el cual deberá referirse a la bibliografía específica si desea realizar un correcto análisis de fotografías hemisféricas.

\section{REFERENCIAS}

Cescatti, A. 2007. Indirect estimates of canopy gap fraction based on the linear conversion of hemispherical photographs. Methodology and comparison with standard thresholding techniques. Agricultural and Forest Meteorology $143(1-2): 1-12$.

Chapman, L. 2008. An introduction to "upside-down" remote sensing. Progress in Physical Geography 32(5):529-542.

Chen, J.M., A. Govind, O. Sonnentag, Y. Zhang, A.G. Barr y B. Amiro. 2006. Leaf area index measurements at FluxnetCanada forest sites. Agricultural and Forest Meteorology 140(1-4):257-268.

Chen, J.M., P.M. Rich, S.T. Gower, J.M Norman y S. Plummer. 1997. Leaf area index of boreal forests: theory, techniques, and measurements. Journal of Geophysical Research 102(D24): 29429-29443.

Cruz-leyva, I.A., J.R. Valdez-lazalde, G. Ángeles-Pérez, H.M. de los Santos-Posadas. 2010. Modelación espacial de área basal y volumen de madera en bosques manejados de Pinus patula y P. teocote en el ejido Atopixco, Hidalgo. Madera y Bosques 16(3):75-97.

Gonsamo, A., J.M.N. Walter y P. Pellikka. 2010. Sampling gap fraction and size for estimating leaf area and clumping indices from hemispherical photographs. Canadian Journal of Forest Research 40(8):1588-1603.
Hill, R. 1924. A lens for whole sky photographs. Quarterly Journal of the Royal Meteorological Society 50(211):227235.

Hu, L., Z. Gong, J. Li y J., Zhu. 2009. Estimation of canopy gap size and gap shape using a hemispherical photograph. Trees 23(5):1101-1108.

Jacobson, R. 2010. The manual of photography. 10a ed. Elsevier. Amsterdam. 566p.

Lang, M., A. Kuusk, M. Mõttus, M. Rautiainen y T. Nilson. 2010. Canopy gap fraction estimation from digital hemispherical images using sky radiance models and a linear conversion method. Agricultural and Forest Meteorology $150(1): 20-29$

Leblanc, S.G., J.M. Chen, R. Fernandes, D. Deering y A. Conley. 2005. Methodology comparison for canopy structure parameters extraction from digital hemispherical photography in boreal forests. Agricultural and Forest Meteorology 129(3-4):187-207.

Macfarlane, C., A. Grigg y C. Evangelista. 2007. Estimating forest leaf area using cover and fullframe fisheye photography: Thinking inside the circle. Agricultural and Forest Meteorology 146(1-2):1-12.

Norman, J.M. y G. Campbell. 1989. Canopy structure. In: Pearcy, R., J. Ehleringer, H. Mooney y P. Rundel, eds. Plant physiological ecology: field methods and instrumentation. Chapman and Hall. Nueva York. p:301-325.

Pekin, B. y C. Macfarlane. 2009. Measurement of crown cover and leaf area index using digital cover photography and its application to remote sensing. Remote Sensing 1(4):1298-1320

Rich, P.M. 1990. Characterizing plant canopies with hemispherical photographs. Remote Sensing Reviews 5:13-27.

Ridler, S. y W. Calvard. 1978. Picture thresholding using an iterative selection method. IEEE Transactions on Systems, Man and Cybernetics 8:260-263.

Schneider, D., E. Schwalbe y H.G. Maas. 2009. Validation of geometric models for fisheye lenses. ISPRS Journal of Photogrammetry and Remote Sensing 64(3):259-266.

Van-den-Heuvel, F.A., R. Verwaal y B. Beers. 2006. Calibration of fisheye camera systems and the reduction of chromatic aberration. ISPRS, Dresden. 6p. http://www.isprs.org/pro- 
ceedings/XXXVI/part5/paper/1267_Dresden06.pdf. Visitado en abril 2010

Walter, J.M.N. 2011. CIMES manual: theory. 24p. http://jmnw. free.fr. Visitado en octubre 2011.

Yamamoto, S.I. 2000. Forest gap dynamics and tree regeneration. Journal of Forest Research 5(4):223-229.

Yamamoto, S.I., N. Nishimura, T. Torimaru, T. Manabe, A. Itaya y K. Becek. 2011. A comparison of different survey methods for assessing gap parameters in old-growth forests. Forest Ecology and Management 262(5):886-893.
Zhang, Y., J.M. Chen y J.R. Miller. 2005. Determining digital hemispherical photograph exposure for leaf area index estimation. Agricultural and Forest Meteorology 133: 166-181.

Manuscrito recibido el 6 de marzo de 2012. Aceptado el 12 de agosto de 2013.

Este documento se debe citar como:

Mauro-Díaz, G., J.D. Lencinas y H. del Valle. 2014. Introducción a la fotografía hemisférica en ciencias forestales. Madera y Bosques 20(1):109-117. 